\title{
Quality of life in patients with recurrent aphthous stomatitis treated with a mucoadhesive patch containing citrus essential oil
}

This article was published in the following Dove Press journal:

Patient Preference and Adherence

27 May 2016

Number of times this article has been viewed

\author{
Esma Kürklü-Gürleyen' \\ Merve Ögüt-Erișen' \\ Onur Çakır ${ }^{\prime}$ \\ Ömer Uysal ${ }^{2}$ \\ Gülsüm Ak' \\ 'Department of Oral Surgery, Faculty \\ of Dentistry, Istanbul University, \\ ${ }^{2}$ Department of Biostatistics and \\ Medical Informatics, Faculty of \\ Medicine, BezmiÂlem Vakif University, \\ Istanbul, Turkey
}

Purpose: To assess 1) patient satisfaction of a mucoadhesive biopatch with citrus essential oil and 2) the change in pain severity and the oral health-related quality of life in patients with recurrent aphthous stomatitis.

Patients and methods: Thirty-seven patients with recurrent aphthous stomatitis participated in the study. Baseline records of personal data, ulcer assessment, visual analog scale, and Oral Health Impact Profile-14 were documented. A mucoadhesive patch was applied over the ulcer. Patients were recommended more applications if pain continued. On the fifth day, a post-therapy assessment was made.

Results: The mean visual analog scale scores at baseline and posttreatment were significantly different (7.3 \pm 2.11 and 4.9 \pm 2.6 , respectively; $P=0.001)$. The mean duration of pain reduced after patch application. The mean total Oral Health Impact Profile-14 scores before and after treatment showed a statistically significant difference $(P=0.001)$. In total, $78.4 \%$ of patients reported a considerable improvement in oral functions after treatment $(P=0.008)$.

Conclusion: The mucoadhesive biopatch containing citrus essential oil resulted in satisfying pain alleviation and restoration of oral functions with a significant improvement in the oral health-related quality of life.

Keywords: recurrent aphthous stomatitis, pain, quality of life, essential oil, biopatch, local therapy

\section{Introduction}

Recurrent aphthous stomatitis (RAS) or canker sore is a painful and ulcerative disorder of the oral mucosa. ${ }^{1,2}$ It occurs worldwide and is reported in every populated continent with a prevalence rate of $25 \%$, ranging between $5 \%$ and $60 \%{ }^{2,3}$ It is well recognized that the symptoms caused by recurrent or chronic oral mucosal lesions such as pain during speaking, eating, and swallowing; discomfort; impairment in food and liquid intake; and problems in interpersonal relationships and self-esteem can deeply affect the oral health-related quality of life (OHRQoL) of patients. ${ }^{4,5}$ RAS, the most frequent oral mucosal disease, was shown to cause all of these symptoms with a significant negative impact in patients' OHRQoL indicating significantly high scores. ${ }^{5}$

The common characteristic features of RAS present as recurrent, solitary or multiple, shallow, round or ovoid, and self-limited ulcers with an erythematous, raised margin, and a yellow grayish floor. ${ }^{6}$ Minor aphthous ulcers, by far the most common type, appear as small ulcers $(5-10 \mathrm{~mm})$ and heal spontaneously in 7-14 days. ${ }^{2}$

Etiology of RAS is still unclear and thought to be multifactorial with precipitating factors such as hematologic deficiency, ${ }^{2,7}$ food hypersensitivity, ${ }^{8}$ familial
Correspondence: Esma Kürklü-Gürleyen Department of Oral Surgery, Faculty of Dentistry, Istanbul University, 34093,

Capa, Fatih, Istanbul, Turkey

Tel +90532574 7976

$\mathrm{Fax}+902125312230$

Email ekurklu@istanbul.edu.tr
Patient Preference and Adherence 2016:10 967-973

967 
tendency, ${ }^{1}$ medications, ${ }^{9}$ hormone imbalance,,${ }^{1,2}$ immunologic abnormalities, ${ }^{1,3}$ and imbalance of oral microbiome. ${ }^{10-12}$ Additionally, several studies suggest that stress may serve as a trigger or a modifying factor for RAS. ${ }^{13}$ In modern society, a number of diseases have been on the rise with changing lifestyles or environmental influences including, diet and psychoemotional stress. ${ }^{14}$ Such impact may contribute to the development and prevalence of RAS.

Pain is the major symptom that starts in the first 24 hours as a tingling or mild irritant sensation, and then ascends and peaks during the first 3-4 days. ${ }^{6}$ Although the disease is of self-limiting nature, the pain which leads to impaired oral food and fluid intake, and subsequent dehydration may be debilitating. Recent researches have shown an association between RAS and imbalances of the oral mucosal microbiome. ${ }^{10-12}$ Performance of oral hygiene procedures, which may be hindered during the active phase of RAS, becomes more important. Given the lack of clarity regarding the etiology of RAS, there is no definitive therapy. Treatment is intended to eliminate the etiologic factors and is palliative with a primary goal of pain alleviation, reduction of ulcer duration, and restoration of oral functions. The secondary goals of treatment include reduction in the frequency and severity of the recurrences and maintenance of remission. No specific treatment that has met all these goals is yet available. ${ }^{15}$ Current treatment modalities include, but not limited to, antibiotics, anti-inflammatory drugs, immune modulators, anesthetics, and alternative (herbal) remedies. Although many attempts including topical medications in forms of gels, creams, pastes, ointments, sprays, and rinses have been made with varying success, ${ }^{15,16}$ such forms are inevitably diluted and eliminated from oral mucosa due to the flushing effect of saliva. Hence, the therapeutic efficiency of the agent decreases. A mucoadhesive system provides a long-term contact with the oral mucosa. Being closed systems, these formulations are protected from saliva, the drug concentrations are controlled, and the drug is continuously delivered for few hours. ${ }^{17}$ Bioadhesive materials also isolate the aphthous ulcer from oral microbiota and trauma. ${ }^{18}$ Drug delivery by this route has its own limitations: 1) drugs which are unstable at buccal $\mathrm{pH}$ cannot be administered; 2) eating and drinking may become restricted; 3) possibility of swallowing the dosage form; 4) overhydration may lead to slippery surface and structural integrity of the formulation may get disrupted by this swelling and hydration of the bioadhesive polymers; 5) drugs which irritate the mucosa or have a bitter or unpleasant taste or an obnoxious odor cannot be administered; 6) only drugs with small dose requirement can be administered; 7) only those drugs which are absorbed by passive diffusion can be administered; and 8) drugs contained in the swallowed saliva are lost. Nevertheless, continued research targets to overcome these drawbacks associated with this route. ${ }^{19}$ Natural-sourced medicines have become increasingly popular among consumers who search for natural ways to maintain their health. The natural agents such as citrus oil and magnesium salts, which were incorporated into a bioadhesive patch, were shown to have antibacterial and anti-inflammatory properties when used in combination. Magnesium also has mild anesthetic properties. ${ }^{20}$ We hypothesized that the combination of these agents in a mucoadhesive carrier applied over an aphthous ulcer may be well tolerated and provide an improvement in the OHRQoL of patients. The objective of this study was to assess 1) the patient satisfaction from an over-the-counter mucoadhesive patch with natural active agents (citrus essential oil and magnesium salts) on minor aphthous ulcers and 2) the change in pain severity and the OHRQoL.

\section{Patients and methods}

This study was conducted as a prospective, consecutivegroup, before-after clinical trial at the Department of Oral Surgery, Faculty of Dentistry, Istanbul University. The study was approved by the Institutional Review Board of Istanbul University, and all participants provided written informed consent after having received a comprehensive explanation of study procedures prior to recruitment.

\section{Study population}

The inclusion criteria to participate in the study were: 1) age $>18$ years, 2) have a history of RAS occurring at least twice a year, 3 ) presenting one active ulcer $(<48$ hours duration) measuring no more than $10 \mathrm{~mm}$ in diameter, and 4) ulcers at accessible locations. Patients with 1) systemic diseases; 2) taking systemic antibiotics, nonsteroidal antiinflammatory drugs, immunomodulatory agents, or local medication within 1 month prior to the study; 3) who are pregnant or lactating; and 4) with any known allergies to the substances in the patch were excluded.

\section{Study medication}

A mucoadhesive patch (Canker Cover ${ }^{\circledR}$; StopEver, Istanbul, Turkey) is a disc of $10 \times 2 \mathrm{~mm}$. The disc had been prepared by compression molding of mixed powders of cross-linked polyacrylic acid and hydroxylpropyl cellulose, and absorbed with citrus oil, menthol, xylitol, and carnallite (potassium 
magnesium chloride). The patch is applied over the aphthous ulcer with slight compression for 20 seconds until complete adhesion.

\section{Assessment}

All patients were assessed twice during the study: baseline and posttreatment (fifth day) assessment. At the first visit, the following steps were undertaken: 1) clinical diagnosis was made by an experienced oral specialist focusing on the minor ulcer with a well-demarcated ulcer on the nonkeratinized oral mucosa, 2) a patient form including personal data and ulcer history was filled by the patients, and 3) Visual Analog Scale (VAS) and the Turkish version of the Oral Health Impact Profile-14 (OHIP-14) were ranked by the patients. ${ }^{21}$

The patients were asked to score the severity of pain on a $100 \mathrm{~mm}$ VAS, a $100 \mathrm{~mm}$ line labeled at one end as "no soreness" and the other end as "worst possible soreness". ${ }^{22}$

OHIP-14 is an OHRQoL scale based on a five-point Likert-type scale. For each of the 14 items, patients were asked how often in the previous 4 weeks they had experienced the problem. Responses were coded $0=$ "never", $1=$ "hardly ever", 2="occasionally", 3="fairly often", and 4="very often". Item responses were summed to produce OHIP-14 total score. Total OHIP-14 scores ranged from 0 (no impact) to 56 (all of the oral health problems were experienced very often). High scores indicated a poor OHRQoL.

\section{Treatment}

At the first visit, a package that contained two patches was given to each patient. One of the patches was applied over the ulcer by the clinician. All the patients were given a written instruction on how to apply the patch over the ulcers. All were informed that the mucoadhesive patch would last for 8-12 hours and were recommended one more application if pain continued after the patch totally resolved. Patients were not given any concurrent local or systemic medication during the study period. Side effects were also monitored.

\section{Posttreatment assessment}

At the fifth day of treatment, clinical examination was made by another clinician who was masked to the baseline assessment scores. The final assessment included VAS, OHIP-14, and a questionnaire which consisted of data on duration of pain, ulcer healing, and status of oral functions.

\section{Statistical analysis}

Sample size was calculated using the decrease in OHIP and VAS scores as primary outcome. Theoretically, the range of
OHIP-14 scores is greater than VAS scores. A minimum of 35 individuals was calculated for 5 units decrease with 10 unit standard deviation (SD) in OHIP scores by $80 \%$ power and 95\% confidence level. Background and demographic data were summarized with descriptive statistics which were presented as mean, SD, and percentage. Spearman correlation coefficient was used for determining the relationship between two quantitative variables. Comparison of the averages of two independent groups of samples was analyzed by using Mann-Whitney $U$-test. Paired mean values were compared by Wilcoxon test. The intrarater agreement was calculated by using Cohen's kappa. A kappa coefficient $>0.40$ represents a moderate degree of agreement. ${ }^{23}$ Collected data were analyzed by using SPSS software (SPSS v 19.0; IBM Corporation, Armonk, NY, USA). The level of significance was established at a $P$-value less than 0.05 .

\section{Results}

Forty-one patients with RAS were consecutively enrolled in the study. Among them, four were dropped from the study because of discontinuation for the final assessment. Thirtyseven patients completed the study. Of the 37 patients, 16 $(43.2 \%)$ were females and $21(56.8 \%)$ were males with a mean age of $38.05 \pm 14.7$ years (range: $15-66$ years).

Following the patch application, the onset of pain relief was found as 5.6 \pm 16.5 hours (range: $0-100$ hours). The mean VAS scores at baseline and posttreatment were significantly different (7.3 \pm 2.11 and 4.9 \pm 2.6 , respectively, $P=0.001$ ). The difference in VAS scores was found to be inversely correlated with patients' age ( $P=0.001$, Spearman correlation coefficient $[r s]=-0.541)$. However, no correlation was detected between the difference in VAS scores and sex. The mean duration of pain in patients' previous ulcers and after patch application was $7.7 \pm 4.3$ days and $21.4 \pm 43.8$ hours, respectively. This result revealed a significant decrease.

The mean overall OHIP-14 scores before and after treatment showed a statistically significant difference $(P=0.001)$ (Figure 1). Table 1 summarizes the details of correlations between the change in overall OHIP-14 scores and the onset of pain relief and duration of pain.

At baseline, all patients complained about limitation and discomfort during oral functions. Twenty-nine patients $(78.4 \%)$ reported an improvement in the oral functions after patch application, whereas eight patients $(21.6 \%)$ reported no change. Covering the ulcers with a patch resolved the impaired oral functions ( $P=0.008)$. The change in mean VAS scores before and after treatment showed no correlation with the functional improvement after patch application $(P=0.49)$. 


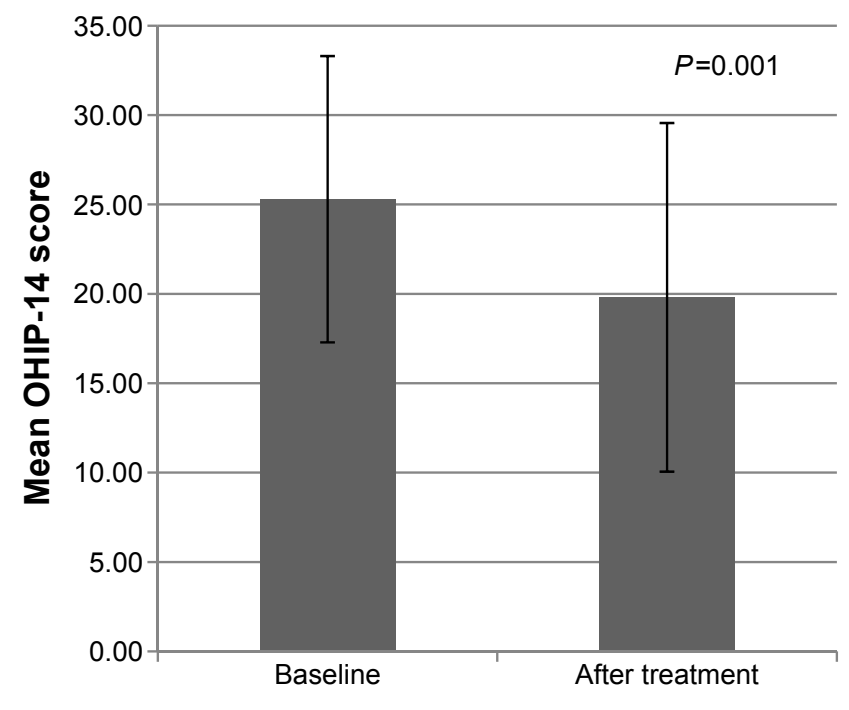

Figure I The mean Oral Health Impact Profile-I4 (OHIP-14) scores at baseline and after treatment.

Similarly, but more interestingly, there was also a lack of correlation between the changes in mean OHIP-14 scores and improvement of oral functions $(P=0.437)$. Seventy-three percent of the patients $(\mathrm{n}=26)$ who reported an improvement in the oral functions claimed a preference for future use. This finding revealed a significant and moderate agreement $(P=0.011$, kappa $=0.41)$.

The patients ranked their satisfaction level for the patch as a mean of $7.05 \pm 2$ over 10 . The size and the adhesiveness of the patch were well tolerated by $64.6 \%$ and $62.2 \%$ of the patients, respectively. In total, $78.4 \%$ of the patients reported positive response for the taste of the patch. Thirty patients $(81.1 \%)$ reported that they would prefer to use the patch for future attacks. A significant and moderate agreement was found between approval of taste and preference of future use $(P=0.011$, kappa $=0.41)$. However, there was no agreement between the "dimension" and "adhesiveness" of the patch and preference of future use. Seventy-three percent of the patients $(n=28)$ claimed to have consciously traumatized the ulcer with their tongue. The onset of pain relief in these patients after patch application was shorter than those who did not traumatize their ulcer. However, the difference was not statistically significant $(P=0.326)$. The change in baseline and posttreatment mean OHIP-14 scores was significantly less in patients who consciously traumatized the ulcer with their tongue than those who did not $(P=0.036)$. The "duration of pain" was not influenced by conscious traumatization $(P=0.667)$.

None of the patients reported any irritation or other side effects at the site of application during patch residence and after its dissolution. Patients declared no further usage for the spare patch.

\section{Discussion}

Given the high prevalence of RAS, dentists and primary care physicians should become familiar with its presentation, related impacts, and management. Patients often report moderate to severe pain when clinical examination reveals only a minor ulcer of $1-2 \mathrm{~mm}$ in diameter. Also, the recurrent pattern and impairment of oral functions make RAS extremely bothersome. Therefore, treatment should primarily focus on pain management, reduction of ulcer duration, and restoration of oral functions. In addition to these, avoiding local traumatic precipitants and preventing secondary infection would enhance the therapeutic outcome. ${ }^{3}$ Therapy for RAS must be directed by the extent of the condition, as determined by the patient and the clinician. Topical treatment is used to promote healing and pain relief, while systemic treatment is reserved for severe cases. ${ }^{16}$ In terms of efficacy, cost, and safety, topical medications remain the treatment of first choice. ${ }^{15}$

Protecting the ulcer from irritating oral fluids and mouth activity is crucial for pain reduction. Therefore, it is desirable to have a drug delivery system that covers and protects the ulcer together with long-term release of medication in the oral cavity. In a study by Shemer et al, a mucoadhesive patch containing citrus oil was found to be more effective than an oral rinse containing benzocaine and benzoin tincture in terms of pain intensity and healing time.${ }^{24}$ In bioadhesive systems prepared by the compression of powders of polyacrylic acid and hydroxypropyl cellulose, the vehicle absorbs moisture when correctly applied and forms a patch which gradually resolves in $\sim 8-12$ hours. Essential oils are secondary metabolites

Table I The correlation between posttreatment ulcer characteristics and change in the overall OHIP scores

\begin{tabular}{lll}
\hline Parameter & $\begin{array}{l}\text { Mean } \pm \text { SD } \\
\text { (posttreatment) }\end{array}$ & $\begin{array}{l}\text { Change in mean overall OHIP scores } \\
\text { between baseline and posttreatment } \\
\text { rs/P (two-tailed)* }\end{array}$ \\
\hline Onset of pain relief (hours) & $5.65 \pm 16.5$ & $-0.014 / 0.933$ \\
Duration of pain (hours) & $21.45 \pm 43.8$ & $-0.078 / 0.647$ \\
\hline
\end{tabular}

Note: *Spearman correlation coefficient.

Abbreviations: OHIP, Oral Health Impact Profile; SD, standard deviation; rs, Spearman correlation coefficient. 
of aromatic plants and in nature serve as antibacterials, antivirals, antifungals, and insecticides. Due to these properties, they are popular in industries of food, cosmetics, and pharmaceuticals. ${ }^{25}$ Citrus lemon essential oil is constituted by two major components (monoterpenes): $\beta$-pinene (13.19\%) and limonene $(70.75 \%)$. The well-recognized antiulcerogenic and gastroprotective activities of essential oils of the genus Citrus are attributable to limonene. The mechanism of these properties is established by its ability to increase mucus secretion, heat-shock protein-70, vasoactive intestinal peptide, and prostaglandin E2. ${ }^{26}$ Citrus essential oil was found to be active against oral pathogens such as Streptococcus mutans, Prevotella intermedia, and Porphyromonas gingivalis. ${ }^{27}$ Based on the contradictory evidence, the contribution of bacterial or viral components to the etiopathogenesis of RAS is nonconclusive. Nevertheless, recent research suggests that the imbalances associated with oral mucosal microbiome, rather than individual infectious pathogens, may play an initiative role for RAS. ${ }^{11}$ It is also indicated that either the presence of a lesion alters the microbiota of the entire oral cavity or a change in microbiota triggers the development of lesions..$^{10}$ As further research is warranted to determine the bacterial diversity over the ulcer site and entire oral mucosa, the antibacterial effect of citrus oil or the barrier property of a mucoadhesive patch on aphthous ulcer remains unclear. The antiulcerogenic effect of limonene might have acted as an immunomodulatory agent for oral aphthous ulcers, though the mechanism should further be investigated.

Magnesium, one of the active ingredients of the patch, has antinociceptive effects in animal and human models of pain. ${ }^{28}$ The significant reduction in pain provided by patch application may be attributable to the presence of magnesium or the coverage of ulcer surface, or a combination of both. Majority of our patients (78.4\%) were satisfied with the taste of the patch most likely due to the presence of menthol in the composition. Menthol is a potent antimicrobial terpene which might cross the cell membranes and penetrate the interior of the cell ${ }^{28}$ and is widely used as a natural product in cosmetics, as a flavoring agent, and as an intermediate in the production of other compounds. ${ }^{29}$

In the present study, $73 \%$ of the patients claimed conscious traumatization of the ulcer with their tongue. Therefore, a close link between the avoidance of trauma by using the patch as a barrier and reduction in pain with improved oral functions may be proposed. In a study by Kutcher et al, mechanical protection to the oral aphthous ulcer was provided by application of tissue adhesives (2-octyl cyanoacrylate). ${ }^{30}$ A considerable pain reduction was demonstrated vs a sham device. In studies which compared the mucoadhesive patch with active ingredient and the placebo, it was concluded that an almost equal effect was found and the mechanical protection of the ulcer alone is important. ${ }^{18}$

The pain management is the backbone of treatment of RAS. Adhesive patch with high patient acceptance was found to be effective in the symptomatic control of pain. The OHRQoL of patients as determined by OHIP-14 revealed a meaningful improvement after treatment with patch. In total, $78.4 \%$ of patients reported a recovery in the oral functions, which revealed a significant difference when compared to baseline values $(P=0.008)$. However, it is interesting to note that improvement in VAS and OHIP-14 scores showed no correlation with functional recovery. This result would be attributed to the inconsistency between two patient-reported outcomes. The taste of the patch and functional recovery appear to be the determinants of patients' preference for future use. The need for repetition of patch application substantially depends on the dissolution period of the patch which in the present study is 8-12 hours. Shemer et $\mathrm{al}^{24}$ recommended once-a-day application of a similar product, whereas in the research by Murray et al, four-times-a-day application of a patch dissolving in $<60$ minutes was the mode of therapy. ${ }^{31}$ Our patients reported no requirement for further use than the two patches. In addition to the aforementioned results, the dimensions of the patch, lack of adverse effects, and once- or twice-daily application probably enhanced the patient compliance.

This study has some limitations which have to be pointed out. The relatively small patient population and lack of comparison to an existing product or matched controls do not allow us to draw any conclusion about the effectiveness of the patch. Standardization with the ulcer severity scoring would add more informative data on the methodology.

Furthermore, as the pain is the common and bothering symptom in RAS, it was our primary goal to investigate the efficacy of the patch on pain relief and impaired oral functions. The results of the study by Sampogna et al on the comparison of patients' and providers' severity evaluation of oral mucosal diseases suggested that the concept of "disease severity" is different from the point of view of patients and of physicians. ${ }^{32}$ Therefore, patient-reported outcomes that measure improvement in patients' quality of life and reduction in morbidity by means of VAS and OHIP-14 scores were undertaken. Nevertheless, the lack of objective outcome measures may come out to be a notable limitation of the study and would be taken into consideration for future research. 


\section{Conclusion}

Forming a long-lasting barrier with a mucoadhesive patch that seals and protects oral ulcer from trauma and contamination with oral pathogens in addition to the antibacterial, antiulcerogenic, and analgesic properties resulted in satisfying pain alleviation and restoration of oral functions with a significant improvement in the OHRQoL.

\section{Acknowledgment}

This work was supported by the Research Fund of Istanbul University (project number: UDP-8583).

\section{Author contributions}

All authors contributed toward data analysis, drafting and critically revising the paper and agree to be accountable for all aspects of the work.

\section{Disclosure}

The authors report no conflicts of interest in this work.

\section{References}

1. Natah SS, Konttinen YT, Enattah NS, Ashammakhi N, Sharkey KA, Häyrinen-Immonen R. Recurrent aphthous ulcers today: a review of the growing knowledge. Int J Oral Maxillofac Surg. 2004;33(3): 221-234.

2. Jurge S, Kuffer R, Scully C, Porter SR. Mucosal disease series. Number VI. Recurrent aphthous stomatitis. Oral Dis. 2006;12(1):1-21.

3. Scully C, Porter S. Oral mucosal disease. Recurrent aphthous stomatitis. Br J Oral Maxillofac Surg. 2008;46:198-206.

4. Llewellyn CD, Warnakulasuriya $\mathrm{S}$. The impact of stomatological disease on oral health-related quality of life. Eur J Oral Sci. 2003;111(4): 297-304.

5. Tabolli S, Bergamo F, Alessandroni L, Di Pietro C, Sampogna F, Abeni D. Quality of life and psychological problems of patients with oral mucosal disease in dermatological practice. Dermatology. 2009;218(4): 314-320.

6. Cawson RA, Binnie WH, Barrett AW, Wright JM. Oral Disease. London: Mosby; 2001.

7. Compilato D, Carroccio A, Calvino F, Di Fede G, Campisi G. Haematological deficiencies in patients with recurrent aphthosis. $J$ Eur Acad Dermatol Venereol. 2010;24(6):667-673.

8. Wardhana, Datau EA. Recurrent aphthous stomatitis caused by food allergy. Acta Med Indones. 2010;42(4):236-240.

9. Healy CM, Thornhill MH. An association between recurrent oro-genital ulceration and non-steroidal anti-inflammatory drugs. J Oral Pathol Med. 1995;24(1):46-48.

10. Bankvall M, Sjoberg F, Gale G, Wold A, Jontell M, Ostman S. The oral microbiota of patients with recurrent aphthous stomatitis. J Oral Microbiol. 2014;6:25739.

11. Hijazi K, Lowe T, Meharg C, Berry SH, Foley J, Hold GL. Mucosal microbiome in patients with recurrent aphthous stomatitis. Clin Invest. 2015;94(3):87S-94S.
12. Seoudi N, Bergmeier LA, Drobniewski F, Paster B, Fortune F. The oral mucosal and salivary microbial community of Behcet's syndrome and recurrent aphthous stomatitis. J Oral Microbiol. 2015;7:271-250.

13. Huling LB, Baccaglini L, Choquette L, Feinn RS, Lalla RV. Effect of stressful life events on the onset and duration of recurrent aphthous stomatitis. J Oral Pathol Med. 2012;41(2):149-152.

14. Al-Omiri MK, Karasneh J, Lynch E. Psychological profiles in patients with recurrent aphthous ulcers. Int J Oral Maxillofac Surg. 2012;41(3): 384-388.

15. Barrons RW. Treatment strategies for recurrent oral aphthous ulcers. Am J Health Syst Pharm. 2001;58(1):41-50.

16. Eisen D, Lynch DP. Selecting topical and systemic agents for recurrent aphthous stomatitis. Cutis. 2001;68(3):201-206.

17. Dixit RP, Puthli SP. Oral strip technology: overview and future potential. J Control Release. 2009;139(2):94-107.

18. Meng W, Dong Y, Liu J, et al. A clinical evaluation of amlexanox oral adhesive pellicles in the treatment of recurrent aphthous stomatitis and comparison with amlexanox oral tablets: a randomized, placebo controlled, blinded, multicenter clinical trial. Trials. 2009;10:30.

19. Chinna Reddy P, Chaitanya KS, Madhusudan Rao Y. A review on bioadhesive buccal drug delivery systems: current status of formulation and evaluation methods. Daru. 2011;19(6):385-403.

20. Mizrahi B, Shapira L, Domb AJ, Houri-Haddad Y. Citrus oil and $\mathrm{MgCl}_{2}$ as antibacterial and anti-inflammatory agents. J Periodontol. 2006;77(6):963-968.

21. Mumcu G, Inanc N, Ergun T, et al. Oral health related quality of life is affected by disease activity in Behçet's disease. Oral Dis. 2006;12(2): $145-151$.

22. Scott J, Huskisson EC. Graphic representation of pain. Pain. 1976; 2(2): $175-184$

23. Landis JR, Koch GG. The measurement of observer agreement for categorical data. Biometrics. 1977;33(1):159-174.

24. Shemer A, Amichai B, Trau H, Nathansohn N, Mizrahi B, Domb A. Efficacy of a mucoadhesive patch compared with an oral solution for treatment of aphthous stomatitis. Drugs R D. 2008;9(1):29-35.

25. Sun J. D-Limonene: safety and clinical applications. Altern Med Rev. 2007;12(3):259-264.

26. Moraes TM, Kushima H, Moleiro FC, et al. Effects of limonene and essential oil from Citrus aurantium on gastric mucosa: role of prostaglandins and gastric mucus secretion. Chem Biol Interact. 2009; 180(3):499-505.

27. Miyake Y, Hiramitsu M. Isolation and extraction of antimicrobial substances against oral bacteria from lemon peel. J Food Sci Technol. 2011;48(5):635-639.

28. Pascual-Ramírez J, Gil-Trujillo S, Alcantarilla C. Intrathecal magnesium as analgesic adjuvant for spinal anesthesia: a meta-analysis of randomized trials. Minerva Anestesiol. 2013;79(6):667-678.

29. Farco JA, Grundmann O. Menthol - pharmacology of an important naturally medicinal "cool". Mini Rev Med Chem. 2013;13(1):124-131.

30. Kutcher MJ, Ludlow JB, Samuelson AD, Campbell T, Pusek SN. Evaluation of a bioadhesive device for the management of aphthous ulcers. J Am Dent Assoc. 2001;132(3):368-376.

31. Murray B, Biagioni PA, Lamey PJ. The efficacy of amlexanox OraDisc on the prevention of recurrent minor aphthous ulceration. J Oral Pathol Med. 2006;35(2):117-122.

32. Sampogna F, Söderfeldt B, Axtelius B, et al. Comparison of patients' and providers' severity evaluation of oral mucosal conditions. $J \mathrm{Am}$ Acad Dermatol. 2011;65(1):69-76. 
Patient Preference and Adherence

Dovepress

\section{Publish your work in this journal}

Patient Preference and Adherence is an international, peer-reviewed, open access journal that focuses on the growing importance of patient preference and adherence throughout the therapeutic continuum. Patient satisfaction, acceptability, quality of life, compliance, persistence and their role in developing new therapeutic modalities and compounds to optimize

Submit your manuscript here: http://www.dovepress.com/patient-preference-and-adherence-journ clinical outcomes for existing disease states are major areas of interest for the journal. This journal has been accepted for indexing on PubMed Central. The manuscript management system is completely online and includes a very quick and fair peer-review system, which is all easy to use. Visit http://www. dovepress.com/testimonials.php to read real quotes from published authors. 